\title{
Tratamiento quirúrgico de la epilepsia en pacientes con cavernomas. Revisión bibliográfica
}

\author{
Francisco José Rojas Zalazar ${ }^{1}$ \\ 1 Instituto de Neurocirugía Dr. Alfonso Asenjo.
}

\begin{abstract}
Resumen
Los cavernomas representan un 5-10\% de las malformaciones vasculares encefálicas y espinales. La manifestación clínica más frecuente de los cavernomas supratentoriales son las crisis epilépticas, se estima que el riesgo de recurrencia luego de una primera crisis es de un $94 \%$ y que un $35-40 \%$ de estos pacientes desarrollan epilepsia refractaria. El objetivo de esta revisión es realizar una puesta al día sobre el rol del tratamiento quirúrgico precoz en pacientes con crisis esporádicas y el tratamiento en paciente con epilepsia refractaria. Además, se mencionan las consideraciones en el tratamiento de cavernomas temporales y extratemporales. Para planificar la cirugía es necesario un adecuado estudio preoperatorio, considerar la localización de la lesión, y tiempo de evolución de la epilepsia. El tratamiento quirúrgico ofrece buenos resultados, un $75 \%$ de los pacientes están libres de crisis epilépticas luego de la cirugía, con mejores resultados en pacientes no refractarios a fármacos.
\end{abstract}

Palabras clave: Cavernoma, epilepsia, cirugía.

\begin{abstract}
Cavernous malformation respresents about 5-10\% of vascular malformations of brain and spine. Seizure is the most frecuent clinical manifestation of supratentorial cavernomas, after a first seizure the risk of recurrence is about $94 \%$ and $35-40 \%$ of these patients will develop refractory epilepsy. The aim of the present review is to discuss the role of surgery in patients with early onset seizure and in patients with refractory epilepsy. We also mention pre-surgical considerations in the treatment of temporal and extra-temporal cavernomas. For surgical planning it is important an accurrate preoperative study as well as considering location of the lesion and time of evolution of seizure. Surgery offer good outcome, $75 \%$ of patientes are seizure free after surgery, better results are achieved in non refractory patients.
\end{abstract}

Key words: Cavernous malformation, epilepsy, surgery.

\section{Introducción}

Los cavernomas son malformaciones vasculares caracterizadas por cavernas dilatadas delimitadas por endotelio fino sin parénquima interpuesto, representan un $5-10 \%$ de las malformaciones vasculares encefálicas y espinales ${ }^{1}$. De acuerdo estadísticas del banco de datos Europeo de cerebro y epilepsia, los cavernomas junto a otras malformaciones vasculares representan un $5,6 \%$ de los hallazgos neuro- patológicos en cirugía de la epilepsia ${ }^{2}$. La manifestación clínica más frecuente de los cavernomas supratentoriales son las crisis epilépticas que con mayor frecuencia se presentan en la tercera década de la vida. Se estima que el riesgo de recurrencia luego de una primera crisis es de un $94 \%$ y que un $35-40 \%$ de estos pacientes desarrollan epilepsia refractaria ${ }^{3,4}$. Los cavernomas temporales tienen un mayor riesgo de desarrollar epilepsia fármaco resistente y aún más los cavernomas temporales mesiales. Dada la alta frecuencia de refractariedad de estas lesiones el tratamiento quirúrgico es una opción a considerar, en las últimas publicaciones se ha planteado que el tratamiento quirúrgico precoz ofrece mejores resultados en cuanto a control de crisis ${ }^{4}$. Existen aspectos importantes a considerar al enfrentar esta patología como son el número de lesiones, localización de la lesión, tiempo transcurrido desde inicio de las crisis, tipo de crisis, como también la correlación entre localización de 
la lesión, semiología de la crisis y estudio electroencefalográfico.

\section{Objetivos}

Los objetivos de esta revisión son:

- Revisar a la luz de la literatura actual el rol de la cirugía en el tratamiento de los pacientes con cavernomas y epilepsia de inicio reciente, epilepsia crónica y pacientes refractarios.

- Estudiar las estrategias quirúrgicas en el manejo de los cavernomas extratemporales y temporales.

- Establecer factores pronósticos.

- Revisar los resultados del tratamiento quirúrgico.

\section{Métodos}

Se realiza una revisión de artículos más relevantes publicados en la literatura en los últimos 10 años, en base de datos pubmed y de algunas referencias incluidas en estos. Se utilizaron las palabras claves: cavernoma, epilepsia, cirugía.

\section{Desarrollo}

\section{Presentación clínica}

Las formas de presentación clínica de estas lesiones son la hemorragia, déficit neurológico y crisis epilépticas, siendo esta última el principal síntoma de presentación en un $40-70 \%$ de los cavernomas supratentoriales. Las crisis son con mayor frecuencia focales, con o sin generalización secundaria y un $40 \%$ de estos pacientes desarrollan epilepsia refractaria a fármacos. Los cavernomas se pueden presentar de manera esporádica o familiar, a esta última presentación se han asociado tres genes CCM1, CCM2, CCM3 y se relacionan con mayor frecuencia a cavernomatosis múltiple, más de un $50 \%$ de estos pacientes presenta más de una malformación cavernomatosa ${ }^{4,5}$.

\section{Epileptogénesis}

Los cavernomas potencialmente epileptogénicos son aquellos que se localizan a nivel cortical (neocorteza) o a nivel temporal mesial (arquicorteza) ${ }^{6}$. Existe consenso de que la epileptogenicidad está dada por las microhemorragias subclínicas que presentan estas lesiones, generando depósito de hemosiderina y gliosis en el tejido cerebral circundante. Esto tendría un rol en la formación de radicales libres, en la activación secundaria de receptores de canales de calcio y exitotoxicidad mediadas por neurotransmisores como aspartato y glutamato. Además, se han descrito altos niveles de serina, glicina, etanolamina en la periferia de los cavernomas y disminución de la captación de glutamato. Estos trastornos bioquímicos podrían aumentar la excitabilidad, la cual se mantiene en el tiempo por los depósitos de hierro en el tejido cerebral. Por otro lado, se ha descrito el rol epileptogénico de otros derivados de la sangre como la albúmina y el depósito de esta en los astrocitos. La exposición continua a actividad eléctrica puede estimular a la generación de sitios de epileptogénesis distantes a la lesión, fenómeno llamado epileptogénesis secundaria ${ }^{4,7,8}$, que ocurriría con mayor probabilidad cuando la lesión se encuentra en o cercana a estructuras que forman parte del sistema límbico, como por ejemplo la región temporal mesial.

\section{Definición de epilepsia asociada a cavernomas y categorías de epilep- sia de acuerdo a la severidad}

La Liga Internacional Contra la Epilepsia (ILAE) define tres tipos de asociación entre cavernoma y epilepsia ${ }^{5}$ :

- Epilepsia relacionada a cavernoma: Epilepsia en pacientes con al menos un cavernoma en que se evidencia el inicio de las crisis en la vecindad del cavernoma.

- Epilepsia probablemente relacionada a cavernoma: Epilepsia en pacientes con al menos un cavernoma y presencia de epilepsia focal que se origina en el mismo hemisferio, pero no necesariamente en vecindad con la lesión.

- Epilepsia no relacionada a cavernoma: Epilepsia en pacientes con al menos un cavernoma, en que no existe relación causal entre cavernoma y epilepsia.

Existen múltiples series sobre cirugía de epilepsia en cavernomas y en muchas de estas, no se distingue entre pacientes con epilepsia farmacorresistente y otras formas de epilepsia menor como crisis ocasionales o epilepsia tratable. En una revisión de Von der Brelie y cols ${ }^{9}$, se revisaron 27 estudios publicados sobre cavernoma y cirugía de la epilepsia con un total de 1.513 pacientes, en un $42,3 \%$ de los casos la categorización sobre el tipo de epilepsia no era clara. En esos estudios los resultados de las series no son útiles para comparar resultados postoperatorios, ya que se mezclan distintas poblaciones independientemente de la severidad de la epilepsia. Algunos autores clasifican tres categorías de epilepsia de acuerdo a la severidad ${ }^{10}$ :

1. Epilepsia farmacorresistente: Persistencia de crisis pese a un tratamiento adecuado con al menos dos fármacos en monoterapia o asociados.

2. Epilepsia crónica: Pacientes que presentan crisis recurrentes sin cumplir los criterios de farmacorresistencia.

3. Crisis esporádicas: aquellos pacientes que han tenido una o dos crisis.

\section{Candidatos a estudio preoperatorio}

El riesgo de presentar crisis en un paciente con cavernoma que sangra y presenta déficit neurológico es de un $6 \%$ y el riesgo de crisis con un cavernoma incidental es de un $4 \%$, en este grupo de pacientes no existe indicación de inicio de tratamiento con fármacos antiepilépticos (FAEs) ${ }^{4}$.

Luego de la primera crisis el riesgo de recurrencia es de un $94 \%$, en estos casos se justifica el inicio de FAEs ${ }^{3,4}$.Por lo tanto, en todo paciente que presente una primera crisis epiléptica y en que el estudio con resonancia magnética (RM) muestre un cavernoma debe quedar con FAEs. Muchos autores están a favor del manejo conservador con anticonvulsivantes en pacientes con una crisis. Sin embargo, aún es materia de debate definir si se justificaría ofrecer tratamiento quirúrgico en pacientes con crisis únicas o crisis recurrentes controladas con FAEs. Al momento de plantear cirugía en estos casos se debe considerar además factores como, localización, riesgo de resangrado, efectos adversos a largo plazo de los FAEs, costos asociados y mala adherencia a tratamiento ${ }^{5}$. Además hay que tener en cuenta que el control de las crisis epilépticas es mejor con cirugía precoz. En un estudio retrospectivo de Dammann $\mathrm{P}$ y cols ${ }^{11}$ en pacientes con epilepsia de diagnóstico reciente, definida como 3 o menos crisis, se observó que el grupo sometido a tratamiento quirúrgico precoz tenía 6,7 veces mayores probabilidades de estar libre de crisis 
y posteriormente libre de FAEs, esto comparado con el grupo conservador que recibió solo manejo farmacológico. De acuerdo a las recomendaciones para el manejo de la epilepsia asociada a cavernomas de la ILAE publicadas el año $2013^{5}$, los pacientes con cavernoma y epilepsia farmacorresistente deben ir a estudio prequirúrgico. Por otro lado, no es necesario aplicar los criterios de farmacorresistencia tradicionales para decidir tratamiento quirúrgico, la falla con monoterapia adecuada puede ser indicación de evaluación preoperatoria. Los pacientes con crisis aisladas con registro interictal y clínica que se correlaciona con la lesión podrían ser candidatos a cirugía.

\section{Estudio preoperatorio}

El objetivo del estudio preoperatorio es determinar la zona epileptogénica, este estudio incluye resonancia magnética de encéfalo (RM) con protocolo de epilepsia, monitorización con video electroencefalografía (Video-EEG) y evaluación neuropsicológica. Este estudio se puede complementar con tomografía computada por emisión de positrones (PET CT) y RM funcional ${ }^{4}$. En un 17,5 a $28 \%$ de los casos es necesario realizar estudio invasivo (fase II), el cual está indicado cuando no es posible identificar la zona epileptogénica, cuando la zona epileptogénica está en relación a áreas elocuentes, ante sospecha de extensión de la zona epileptogénica fuera de la lesión o en presencia de cavernomas múltiples con foco no concluyente. El estudio invasivo puede ser realizado mediante la instalación de electrodos subdurales (grillas) o electrodos profundos. La selección de los pacientes para cirugía está basada en la congruencia entre los hallazgos electroclínicos, localización de la lesión en la RM y estudios complementarios como RM funcional para estudio de lateralidad de lenguaje y memoria o PET CT que puede mostrar zonas de hipometabolismo interictal que se correlacionan con la zona epileptogénica ${ }^{12}$.

\section{Tratamiento quirúrgico}

Las alternativas quirúrgicas para el tratamiento de estas lesiones son la lesionectomía, lesionectomía más resección del anillo de hemosiderina, lesionectomía con resección del anillo de hemosiderina y corteza epileptogénica (lesionectomía extendida o lobectomía) $)^{4,5,8,10}$.
Debido a que los cavernomas no contienen tejido neuronal, no podrían ser considerados exclusivamente como zona epileptógena, de manera que el tratamiento quirúrgico debe estar ligado a los efectos que genera en el tejido cerebral circundante. Aunque existen resultados dispares, la mayoría de los estudios reportan mejores resultados en cuanto a control de crisis cuando se reseca el anillo de hemosiderina ${ }^{4,5,13,14}$. Con el tiempo ha surgido una estrategia quirúrgica más bien orientada a la epilepsia que a la lesión, lo que implica realizar resecciones teniendo en cuenta factores como localización de la lesión (temporal, extratemporal), tiempo de evolución de la epilepsia, hallazgos en estudios complementarios como PET CT, RM funcional o el uso de electrocorticografía intraoperatoria. Hay que considerar que la lesionectomía sería menos efectiva en pacientes con epilepsia de mayor duración y de mayor severidad $^{15}$

Para planificar la cirugía es necesario distinguir entre cavernoma único o múltiples. En este último caso los hallazgos electroclínicos orientan a cuál lesión resecar, y cuando el foco epilep- tógeno no es claro, es necesario realizar un estudio invasivo. Otro aspecto fundamental es distinguir si se trata de un cavernoma temporal o extratemporal. En el caso de los cavernomas extratemporales la recomendación es realizar lesionectomía y resecar el anillo de hemosiderina (Figura 1).

En el caso de lesiones neocotricales temporales, se recomienda la lesionectomía, a menos que existan marcadores de epileptogenicidad, como anomalías estructurales del hipocampo y epilepsia de larga data. Cuando la lesión se encuentra en relación a área elocuente se sugiere el mapeo cortical con grillas, o mapeo intraoperatorio motor o de lenguaje ${ }^{15}$ (Figura 2). El uso de electrocorticografía intraoperatoria como medida para mejorar los resultados quirúrgicos en cuanto a control de crisis es controversial, existen estudios controlados pequeños que sugieren que la electrocorticografía intraoperatoria podría ofrecer mejores resultados en el control de crisis, sin embargo, de acuerdo a recomendación de expertos su uso no es estrictamente necesario $^{5,9,17}$

Para planificar el tratamiento quirúrgi-

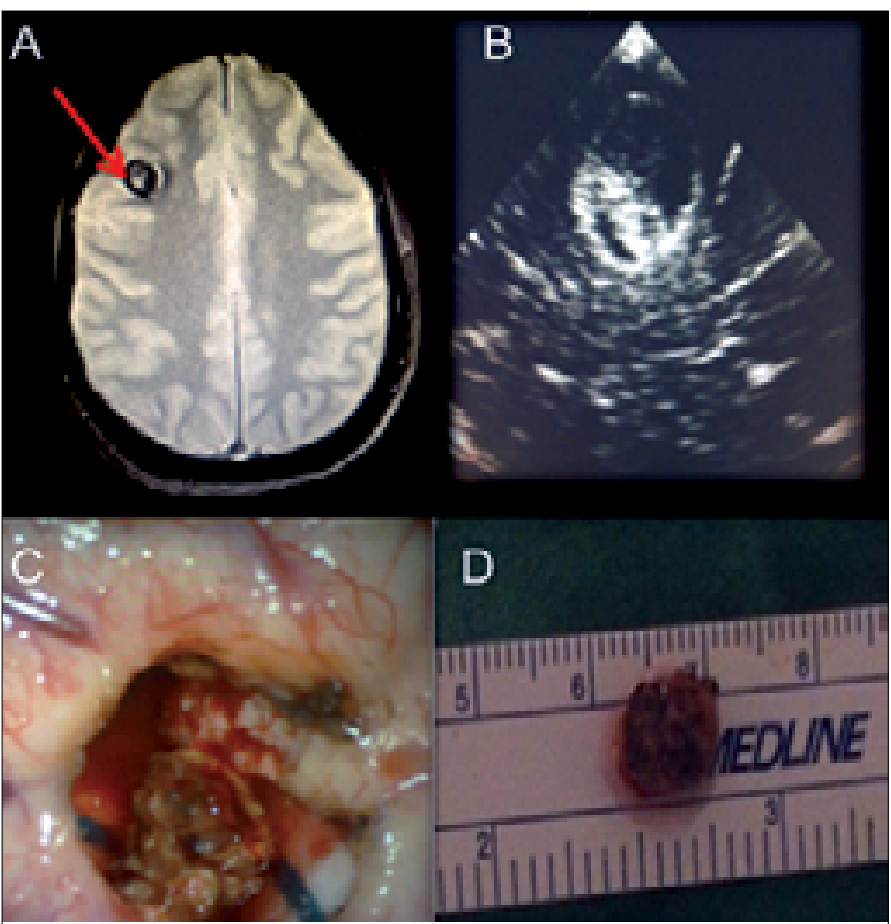

Figura 1. A) RM T2 gradiente corte axial. Cavernoma frontal superior derecho con artefacto de suceptibilidad magnética ("blooming") y anillo de hemosiderina en la periferia; B) Ecografía intraoperatoria; C) Resección del cavernoma y anillo de hemosiderina. Se observa el cavernoma en el centro; D) Cavernoma pieza macroscópica. 


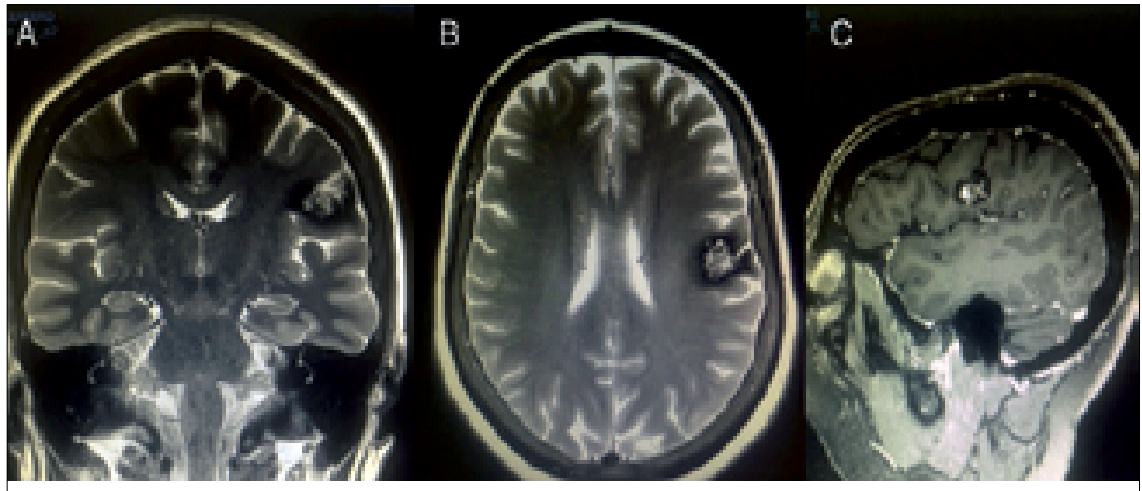

Figura 2. A) Secuencia T2 coronal; B) Axial; C) Sagital. Paciente de 59 años con epilepsia farmacorresistente, crisis focales con clonias faciales derechas y disartria. RM de encéfalo muestra cavernoma frontal inferior derecho en contacto con el área motora. Presenta morfología de "palomita de maíz", contornos lobulados, marginada por anillo de hemosiderina y área central con distintas intensidades de señal.

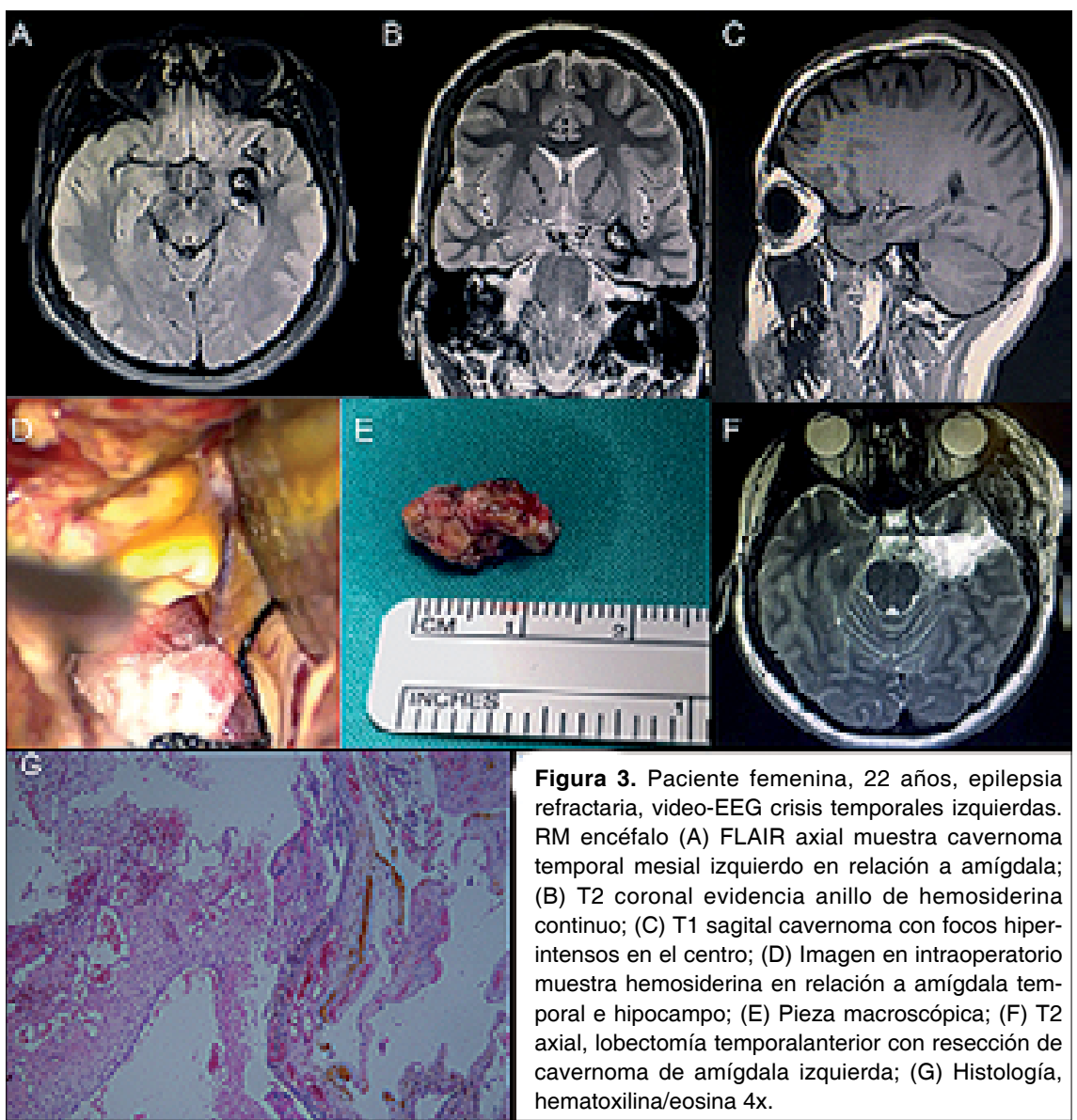

co de los cavernomas temporales es necesario distinguir si está localizado a nivel mesial o en relación a la neocorteza, definiendo como límite entre ambas regiones el surco colateral. Los cavernomas temporales tienen mayor riesgo de desarrollar farmacorresisten- rosis temporal mesial, displasia cortical) y/o con epilepsia de larga duración se recomienda la lobectomía tempo$\mathrm{ral}^{1,4,5,15,18,19}$. Sin embargo, en algunas series como la de Yong- Shi y cols, Okishev y cols ${ }^{20,21}$ realizan resecciones más selectivas que incluyen lesionectomía y resección de amígdala e hipocampo, estos autores utilizan electrocorticografía intraoperatoria.

Cuando la lesión se encuentra entre la neocorteza y la región temporal mesial, es necesario tener en cuenta algunos factores para decidir si se reseca de manera adicional amígdala e hipocampo, estos son: proximidad del cavernoma a la región temporal mesial, tiempo de duración de la epilepsia, alteraciones en RM (esclerosis hipocampal) o hipometabolismo en esta región en el estudio con PET-CT ${ }^{18}$. Además es fundamental considerar la evaluación neurocognitiva, lateralidad de lenguaje y memoria, para predecir el potencial deterioro del paciente en el postoperatorio. En el estudio de Jehi L y cols. ${ }^{1}$, se observó mejor control de crisis en pacientes con cavernomas temporales tratados con lobectomía temporal, además los subgrupos de pacientes con mayor duración de la epilepsia tuvieron mejor control de crisis con lobectomía temporal que el grupo sometido a lesionectomía (Figura 3). En la Figura 4 se presenta un algoritmo de manejo propuesto para el tratamiento quirúrgico de los cavernomas asociados a epilepsia.

\section{Cavernomas múltiples}

La presencia de más de una lesión potencialmente epileptogénica ha sido descrita como factor de riesgo de mal control de crisis. Cuando se considera la opción quirúrgica es necesario un adecuado estudio preoperatorio para identificar la lesión epileptogénica, en caso de no existir congruencia entre clínica, localización de la lesión y video electroencefalograma, es necesario el estudio invasivo. En la serie de Von der Brelie y cols. ${ }^{22}$, un $78 \%$ de los pacientes no requirió estudio invasivo, mientras que en la serie de Rocamona y cols..$^{23}$ solo 1 de 10 pacientes tratados requirió estudio invasivo previo a la cirugía. Cuando se logra identificar una lesión epileptogénica aislada, la cirugía ofrece buenos resultados, en diferentes series de cavernomas múltiples el porcentaje de pacientes libre de crisis varía entre un $70-81 \%^{22,23,24}$. 


\section{Epilepsia asociada a cavernoma: tratamiento quirúrgico}

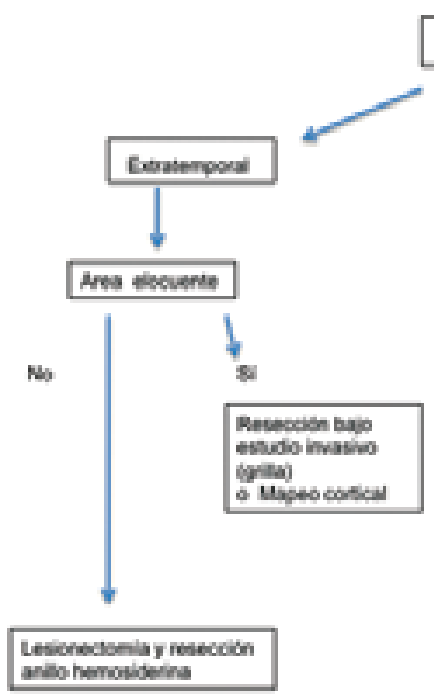

Cavernem

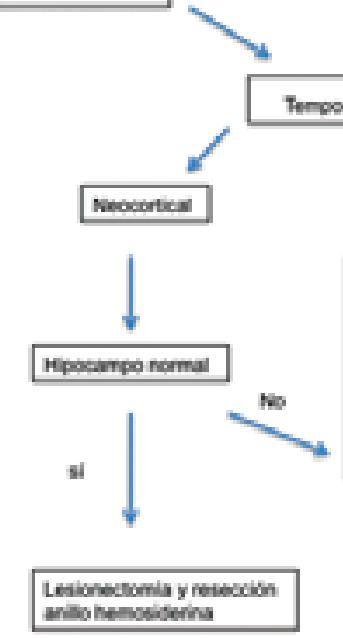

Figura 4. Esquema de manejo propuesto para el tratamiento quirúrgico de la epilepsia asociada a cavernomas.

\section{Control de crisis post tratamiento quirúrgico}

La cirugía ofrece buenos resultados en términos de control de crisis. En uno de los estudios multicéntricos más grandes sobre resultados postoperatorios en pacientes con cavernomas de Baumman y cols. ${ }^{25}$, que incluyó 168 pacientes todos con más de 3 crisis previo a la cirugía, el $70 \%$ de los pacientes estaba clasificado en Engel I y $68 \%$ de los pacientes persistía libre de crisis con seguimiento a 2 años. Englot y cols. ${ }^{26}$, revisaron 31 estudios incluyendo 1.226 pacientes, el $75 \%$ de los pacientes tratados con cirugía quedó libre de crisis epilépticas (Engel I) con un seguimiento mínimo de 6 meses. En un estudio retrospectivo de Know $\mathrm{C}$ y cols. ${ }^{27}$, que incluyó 56 pacientes un $82,1 \%$ de los pacientes quedó libre de crisis epilépticas. En uno de los estudios más grandes de cirugía de epilepsia asociada a cavernoma en pacientes pediátricos publicado por Von der Brelie y cols. ${ }^{28}$, se observó que en el grupo con epilepsia de menos de 2 años de evolución, el $100 \%$ de los pacientes quedó libre de crisis epilépticas (ILAE I) mientras que en el grupo de pacientes con más de 2 años de evolución el 63,6\% quedó libre de crisis epilépticas. En el estudio retrospectivo de Van Gompel y cols. ${ }^{29}$, sobre evaluación de satisfacción de los pacientes postoperados de cavernomas con epilepsia asociada, el $87 \%$ es- taba libre de crisis con un seguimiento promedio de 97 meses, además el $90 \%$ aceptaría nuevamente el tratamiento quirúrgico.

\section{Factores pronósticos que afectan el control de crisis en el postoperatorio} Se ha descrito una correlación negativa entre el tiempo transcurrido entre el inicio de la epilepsia y el tratamiento quirúrgico, cirugías más tardías tendrían peores resultados en término de control de crisis ${ }^{11,27,30,31,32,33}$. En un estudio retrospectivo de Dammann y cols. ${ }^{11}$, que comparó los resultados del tratamiento conservador vs tratamiento quirúrgico en 79 pacientes con epilepsia de reciente comienzo (3 o menos crisis), se observó que las posibilidades de estar libre de crisis (ILAE I) a 5 años en el grupo tratado con cirugía precoz (menos de 6 meses de evolución) era de un $73 \%$, en el grupo tratado con fármacos un $22 \%$ de los pacientes estaba libre de crisis y en el grupo de pacientes tratados con cirugía de manera tardía (más de 12 meses de evolución) un $68 \%$ estaba libre de crisis. Además en el grupo que recibió tratamiento quirúrgico precoz un $71 \%$ de los pacientes quedó libre de crisis y de fármacos. En el estudio retrospectivo de Kim J y cols. $^{33}$, que incluyó 46 pacientes divididos en dos grupos, uno con crisis esporádicas y el segundo con epilepsia crónica, se compararon los resultados del tratamiento quirúrgico con seguimiento a 8 años, el porcentaje de pacientes Engel I a 8 años era de un $100 \%$ y $90 \%$ respectivamente.

Otros factores asociados a mal pronóstico son la resección incompleta del anillo de hemosiderina, mayor frecuencia de crisis, crisis generalizadas, lesiones múltiples y cavernomas mayores a $1,5 \mathrm{~cm}^{15,26,32,33}$.

\section{Morbilidad y estado cognitivo post operatorio}

La mayoría de las series reporta resultados en términos de control de crisis pero no se reporta de manera sistemática la morbilidad y estado cognitivo postoperatorio. En la serie de Cossu y cols. ${ }^{4}$, que incluyó 40 pacientes de los cuales 20 fueron sometidos a lesionectomía y 20 a lesionectomías extendidas se reportó un 7,5\% (3/40) de déficit postoperatorio permanente, reportándose un caso de compromisos de memoria verbal y visuoespacial luego de lobectomías temporal izquierda, un caso de disfasia y hemianopsia derecha luego de lobectomía temporal izquierda y un caso compromiso motor de la mano derecho luego de una lesionectomía en región Rolándica derecha. En la serie de cavernomas temporales de Kielev y cols. ${ }^{34}$, se reportó un $10 \%$ (4/40) de déficit de memoria postoperatorio luego de lesionectomías.

\section{Discusión}

Evidencia disponible en la literatura La mayoría de los trabajos publicados sobre epilepsia asociada a cavernomas corresponden a estudios retrospectivos, series de casos y revisiones sistemáticas. Existe disparidad en las publicaciones en cuanto a criterios para clasificar el tipo de epilepsia, lo cual podría explicar diferentes resultados en las series en cuanto a control de crisis, factores pronósticos y estrategias quirúrgicas. Las escalas para medir control de crisis y el tiempo de seguimiento de los pacientes varía en diferentes estudios, lo cual también limita la comparación de resultados. La mayoría de las series revisadas reporta resultados en términos de control de crisis postoperatoria, pero no se hace mención a resultados cognitivos ni de calidad de vida. Dado lo anterior existe la necesidad de nuevos estudios que diferencien grupos de pacientes según 
el tipo de epilepsia, estudios prospectivos, evaluación cognitiva postoperatoria y de calidad de vida ${ }^{4,9,18,30}$.

\section{Cirugía precoz}

En la mayoría de los estudios se describe un mejor control de crisis en pacientes operados de manera precoz, por lo cual cuando existe un cavernoma asociado a epilepsia, se debe considerar la resección quirúrgica precoz y especialmente cuando existe epilepsia refractaria ${ }^{5,35,36}$, dados los mejores resultados con cirugía precoz no es necesario esperar la farmacorresistencia para realizar un tratamiento quirúrgico. Sin embargo, aún es materia de discusión plantear tratamiento quirúrgico en pacientes con cavernomas y crisis única. Varias series reportan mejor control de crisis en pacientes con epilepsia de menos de 1 año de evolución, sin embargo, aún no existe consenso sobre cuán precoz debe ser la cirugía.

Esperar a la refractariedad expone a los pacientes a peores resultados en términos de control de crisis, a tratamientos prolongados con FAEs, riesgos de efectos adversos, deterioro cognitivo y además al riesgos de desarrollar epileptogénesis secundaria ${ }^{8}$.

\section{Estrategia quirúrgica: lesionectomía vs lesionectomía extendida}

Existe consenso que en el momento de planificar la cirugía se debe considerar la localización de la lesión. Cuando el cavernoma se encuentra a nivel extra temporal se recomienda la lesionectomía con resección del anillo de hemosiderina, resección que se puede ver limitada en lesiones en área elocuente para preservar función. Los pacientes con cavernomas temporales presentan un mayor riesgo de desarrollar epilepsia refractaria y epileptogénesis secundaria, en particular aquellos localizados a nivel temporal mesial. En este grupo de pacientes se aconseja realizar lesionectomías extendidas que incluyan las estructuras temporales mesiales, lo cual se asocia a mejores resulta$\operatorname{dos}^{1,15,18,19,20,21}$. En las series revisadas de pacientes con cavernomas temporales que requirieron resecciones de estructuras temporales mesiales o lobectomía temporal existe escasa mención del estado neurocognitivo postoperatorio de los pacientes ${ }^{1,10,15,18,19,20,21,34}$.

El impacto de la cirugía en la función neurocognitiva del paciente es un punto esencial en cirugía de la epilepsia y de particular relevancia en epilepsia temporal, por lo cual, se debe considerar el potencial riesgo de deterioro neuropsicológico postoperatorio en base a la evaluación neuropsicológica preoperatoria, que se puede complementar con RM funcional para determinar lateralidad de lenguaje y memoria. Pacientes con mejor estado neurocognitivo preoperatorio presentan mayor riesgo de deterioro cognitivo postquirúrgico, situación que debe ser considerada en conjunto con el paciente al momento de definir el tipo de cirugía como es el caso de la lobectomía temporal ${ }^{4,19,34,37}$.

El control de crisis postoperatorio no se ve afectado por la localización de la lesión temporal o extra temporal ${ }^{35}$, ni existen tampoco diferencias de control de crisis postoperatoria entre cavernomas temporales neocorticales y mesiales ${ }^{18}$.

\section{Cavernomas múltiples}

La presencia de cavernomas múltiples no excluye la posibilidad de un tratamiento quirúrgico, cuando el cavernoma responsable de las crisis está identificado, los resultados pueden ser comparables a series con cavernomas únicos.

\section{Conclusiones}

En los pacientes con epilepsia asociada a cavernoma la probabilidad de desarrollar farmacorresistencia es alta. Luego de un estudio preoperatorio adecuado la cirugía ofrece buenos resultados en términos de control de crisis. La tendencia actual es a no esperar criterios estrictos de farmacorresistencia para indicar cirugía y cuando es precoz la probabilidad de lograr la libertad de crisis es mayor, por lo cual los pacientes con crisis de reciente comienzo o esporádicas también pueden ser considerados candidatos a cirugía. La estrategia quirúrgica de los cavernomas asociados a epilepsia está dada por tiempo de evolución de la epilepsia, hallazgos adicionales en la RM como por ejemplo patología dual, estado neurocognitivo previo del paciente $y$ localización de la lesión temporal, extratemporal o área elocuente.

\section{Trabajo de Ingreso Sociedad de Neurocirugía}

Recibido: 08 de diciembre de 2018 Aceptado: 26 de diciembre de 2018

\section{Referencias}

1. Jehi LE, Palmini A, Aryal U, Coras R, Paglioli E. Cavernous malformation in the setting of focal epilepsies: pathological findings, clinical characteristics and surgical treatment principles. Acta Neuropathol. 2014;128:55-65.

2. Blümcke I, Aronica E, Urbach H, Alexopoulos A, González-Martínez J. A neuropathology-based approach to epilepsy surgery in brain tumors and proposal for a new terminology use for long-term epilepsy-associated brain tumors. ActaNeuropathol. 2014;128:39-54.

3. Josephson CB, Leach JP, Duncan R, Roberts RC, Counsell CE, Al- Shahi Salman R. Seizure risk from cavernous or arteriovenous malformations: prospective population-based study. Neurology. 2011;76:1548-54.

4. Cossu M, Raneri F, Casaceli G, Gozzo F, Pellicia V, Lo Russo G. Surgical treatment of cavernoma related epilepsy. Neurosurg SCI. 2015;59:237-53.

5. Rosenow F, Alonso-Venegas M, Baumgartner C, Blumke I, Carreño M, Gizewski R, et al. Cavernoma-related epilepsy: Review and recommendations for management-Report of the surgical task force of the ILAE commission on therapeutic strategies. Epilepsia.2013;54 (12): 2025-35.

6. Mezler K, Chen Xu, Thiel P, Iwinska- Zelder J, Miller D, Reuss A, et al. Epileptogenicity of cavernomas depends on (Archi-) Cortical Localization. Neurosurgery. 2010;67(4):918-924.

7. Sevy A, Gavaret M, Trebuchon A, Vaugier L, et al. Beyond the lesion: The epileptogenic networks around cavernous angiomas. Epilepsy research. 2014; 108:701-708. 
8. Komotar, R, Mikell C, McKhann G. Epilepsy Surgery versus Lesionectomy in Patients with Seizures Secondary to Cavernous Malformations. Clinical Neurosurgery.2008;55:101-107.

9. Von der Brelie C, Schramm J. Cerebral cavernous malformation and intractable epilepsy: The limited usefulness of the current literature. Acta Neurochir (Wein). 2011;153:249-259.

10. Von der Brelie, Malter M, Niehusmann P, Elger C, Von Lehe M, Schramm J. Surgical management and long-term seizure outcome after epilepsy surgery for different types of epilepsy associated with cerebral cavernous malformation. Epilepsia. 2013;54(9): 1699-1706.

11. Dammann P, Werde K, Jabbarli R, Neuschulte S, Menzler K, Zhu Y, et al. Outcome after conservative Management or surgical treatment for new onset epilepsy in cerebral cavernous malformation. J Neurosurg. 2017;126:1303-1311.

12. Englot $D$. A modern epilepsy surgery treatment algorithm: incorporating traditional and emerging Technologies. Epilepsy and behavior. 2018;80:68-74.

13. Dammann P, Schaller C, Sure U. Should we resect perilesional hemosiderin deposits when performing lesionectomy in patients with cavernoma -related epilepsy (CRE). Neurosurg Rev. 2017; 40:39-43.

14. Baumnn C, Schucknecht B, Lo Russo G, Cossu M, Citterio A, Andermann F, et al. Seizure Outcome after Resection of Cavernous Malformations Is Better When Surrounding Hemosiderin-stained Brain Also is Removed. Epilepsia. 2006; 47(3):563-566.

15. Upchurch K, Stern, J, Noriko S, Dewar S, Engel J, Vinters, H, et al. Epileptogenic temporal cavernous malformations: operative strategies and postoperative seizure outcomes. Seizure. 2010;19:120-128.

16. Zanello M, WagerM, Corns R, Capelle I, Mandonnet E, Fontaine D, et al. Resection of cavernous angioma located in eloquent areas using functional cortical and subcortical mapping under awake conditions. Outcome in a 50- case multicenter series. Neurochirurgie. 2017; 63: 219-226.

17. San Juan D, Díaz-Núñez I, Ojeda-Baldéz, Barajas-Juárez A, González-Hernández I, Alonso-Venegas M, et al. Utility of electrocorticography in the surgical treatment of cavernomas presenting with pharmacoresistant epilepsy. Epileptic Disord. 2014;168(3):245-260.

18. Yang PF, Pei JS, Jia YZ, Lin Q, Xiao H, Zhang T, et al. Surgical Management and long - term seizure outcome alter surgery for temporal lobe epilepsy associated with cerebral cavernous malformations. World Neurosurgery. 2018.110;659-670.

19. Vale F, Vivas C, Manwaring J, Schoemberg M, Benbadis S. Temporal lobe epilepsy and cavernous malformations: surgical strategies and long- term outcomes. Acta Neurochir. 2015;157:1887-1895.

20. Shan YZ, Fan XT, Meng L, An Y, Xu JK, Zhao GG. Treatment and outcome of epileptogenic temporal cavernous malformations. Chinese Medical Journal. 2015;128:909-913.

21. Okishev DN, Belousova OB, Shekhtman OD, Eliava SS, Sazonova OB, Kopachev DN. Amygdalohippocampectomy in treatment of epilepsy in patients with temporal lobe cavernomas. Zh Vopr Neirokhir Im N Burdenko. 2016;80(1):35-43.

22. Von der Brelie C, Von Lehe M, Raabe A, Niehusman P, Urbach H, Meyer C, et al. Surgical resection can be successful in a large fraction of patients with drug- resistant epilepsy associates with multiple cerebral cavernous malformations.Neurosurgery. 2014;74(2):147-153.

23. Rocamonra R, Mader I, Zetner J, Schulze - Bonhage A. Epilepsy surgery in patients with multiple cerebral cavernous malformations. Seizure. 2009;18(4):241-245.

24. Kivelev J, Niemela M, kivisari R, Dashti R, Laakso A, Hernesniemi J. Long -term outcome of patients with multiple cerebral cavernous malformations. Neurosurgery. 2009;65(3):450-455.

25. Baumann C, Acciarri N, Bertalanffy H, Devinsky O, Elger C, Lo Russo G, et al. Seizure outcome after resection of supratentorial cavernous malformations: A study of 168 patients. Epilepsia. 2007;48(3):559-563.

26. Englot D, Han S, Lawton M, Chan E. Predictors of seizure freedom in the surgical treatment of supratentorial cavernous malformation. Journal of Neurosurgery. 2011;115:1169-1174

27. Kwon C, Sheth S, Walcott B, Neal W, Eskandar E, Ogilvy C. Long - term seizure outcomes following resection of supratentorial cavernous malformations. Clinical Neurology and Neurosurgery. 2103;115:2377-2381.

28. Von der Brelie, Kuczaty S, Von Lehe M, Surgical management and long-term outcome of pediatric patients with different subtypes of epilepsy associated with cerebral cavernous malformations. J Neurosurg Pediatrics. 2014; 13:699-705.

29. Van Gompel J, Marsh R, Meyer F, Worrell G. Patient - assessed satisfaction and outcome after microsurgical resection of cavernomas causing epilepsy. Neurosurg Focus. 2010. 29(3):E16.

30. Ferroli $\mathrm{P}$, Casazza M, Marras $\mathrm{C}$, et al. Cerebral cavernomas and seizures: a retrospective study on 163 patients who underwent pure lesionectomy. Neurol Sci 2006;26:390-4.

31. Wang C, Yu X, Shresth S, Qian C, Wang I, Chen G. A predicted model for postoperative seizure outcomes after the surgical resection of supratentorial cavernous malformations. Medicine( Baltimore). 2016;95 (26):e4078.

32. Kim W, Stramtas S, Choy W, Dye J, Nagasawa D, Yang I. Prognostic factors for post-operative seizure outcomes after cavernous malformation treatment. Journal of Clinical Neuroscience. 2011;18:877-880.

33. Kim J,Kim C, Chung C. Longitudinal changes in seizure outcomes after resection of cerebral cavernous malformations in patients presenting with seizures: along - term follow-up of 46 patient. Acta Neurochir. 2014;156:1539-1547.

34. Kivelev J, Niemelä M, Blomstedt G, Roivainen R, Lehecka M, Hernesniemi J. Microsurgical treatment of temporal lobe cavernomas. Acta Neurochir (Wien). 2011;153:253-270.

35. Kangmin H, Shize J, Jianping S, Zehan W, Liang C Ying M. Long-Term Outcomes of Surgical Treatment in 181 Patients with Supratentorial Cerebral Cavernous Malformations Associated Epilepsy. World Neurosurgery. 2017;108:869-875.

36. Akers A, Al-Shahi Salaman R, Awad I,Dahlem K, Flemming K, Hart B, et al. Synopsis of guidelines for the Clinical Management of cerebral cavernous malformations: Consensus recommendations based on systematic literature review by the angioma Alliance advisory board clinical Experts panel. Neurosurgery. 2017;80(5):665-680.

37. Witt J, Coras R, Schramm J, Becker A,Elger C, Blümcke I, et al. Relevance of hippocampal integrity for memory outcome after surgical treatment of mesial temporal lobe epilepsy. J Neurol. 2015;262(10):2214-2214.

\section{Correspondencia a:}

Dr. Francisco José Rojas Zalazar

Instituto de Neurocirugía Dr. Alfonso Asenjo

José Manuel Infante 553, Providencia, Santiago de Chile.

Teléfono: 56973891504

frojasz@ hotmail.com 